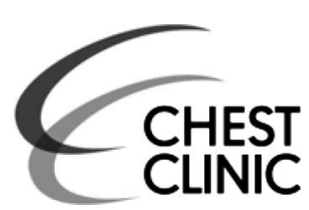

${ }^{1}$ Division of Pulmonary and Critical Care Medicine, Department of Medicine, Albany Medical College, Albany, New York, USA

${ }^{2}$ Division of Pulmonary and Critical Care Medicine, Department of Medicine, Medical University of South Carolina, Charleston, South Carolina, USA

${ }^{3}$ Department of Medicine, Albany Medical College, Albany, New York, USA

\section{Correspondence to}

Dr Amit Chopra, Division of Pulmonary and Critical Care Medicine, Department of Medicine, Albany Medical College, 47 New Scotland Avenue, Albany, NY 12208-3479, USA; chopraa1@mail.amc.edu

Received 10 April 2016 Revised 7 May 2016 Accepted 19 May 2016 Published Online First 4 July 2016

\title{
The value of sound waves and pleural manometry in diagnosing a pleural effusion with the dual diagnosis
}

Amit Chopra, ${ }^{1}$ Rahul Argula, ${ }^{2}$ Christopher Schaefer, ${ }^{3}$ Marc A Judson, ${ }^{1}$ Terrill Huggins ${ }^{2}$

\section{CASE}

An 83-year-old woman presented with a recurrent, right-sided pleural effusion. She had undergone three large volume thoracentesis in the past 2 years, which revealed a 'clear appearing' transudative effusion. She had a remote history of haemothorax, a complication from dual chamber pacemaker placement. A chest radiograph (figure 1A) showed a moderate sized, right pleural effusion. Pleural ultrasonography revealed an anechoic fluid collection. Approximately $1.2 \mathrm{~L}$ of yellow fluid was removed during thoracentesis. Pleural manometry performed during the thoracentesis revealed a biphasic pressure-volume (P-V) curve showing a steep increase in pleural space elastance at the terminal stages of drainage suggesting an unexpandable lung (figure 2). An air-contrast chest CT scan (figure 1B) showed abnormal visceral pleural thickening consistent with a trapped lung. However, given the initial flat portion of the P-V curve in a pre-existing transudate, we surmised that there was an additional cause of the pleural effusion besides the trapped lung. Preprocedural ultrasound scanning prior to thoracentesis showed hydronephrosis of the right kidney, leading us to clinically suspect the presence of an urinothorax. Pleural fluid analysis was consistent with a transudative effusion with a serum/pleural fluid creatinine ratio of 1.1. A $99 \mathrm{Tm}$ radionuclide renal scintigraphy scan demonstrated extravasation of radionuclide into the right pleural space (figure 1C), confirming the presence of an urinothorax.

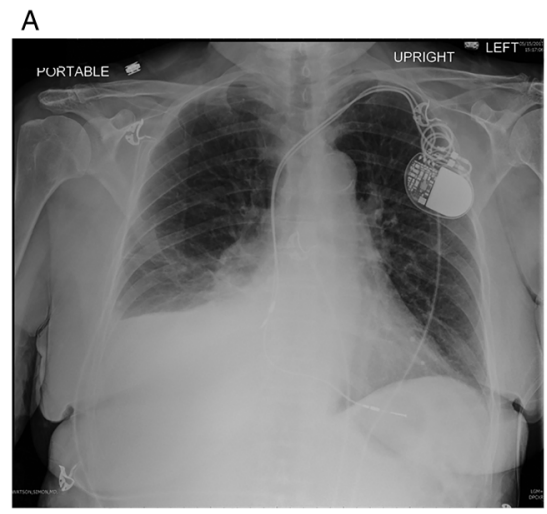

B

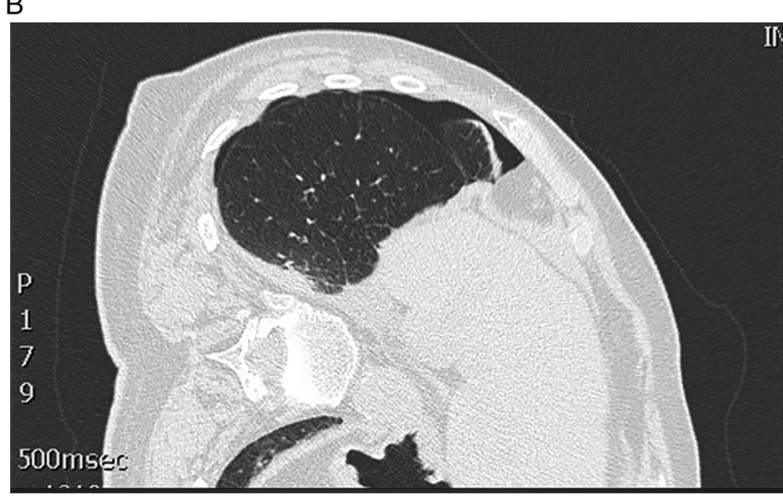

C

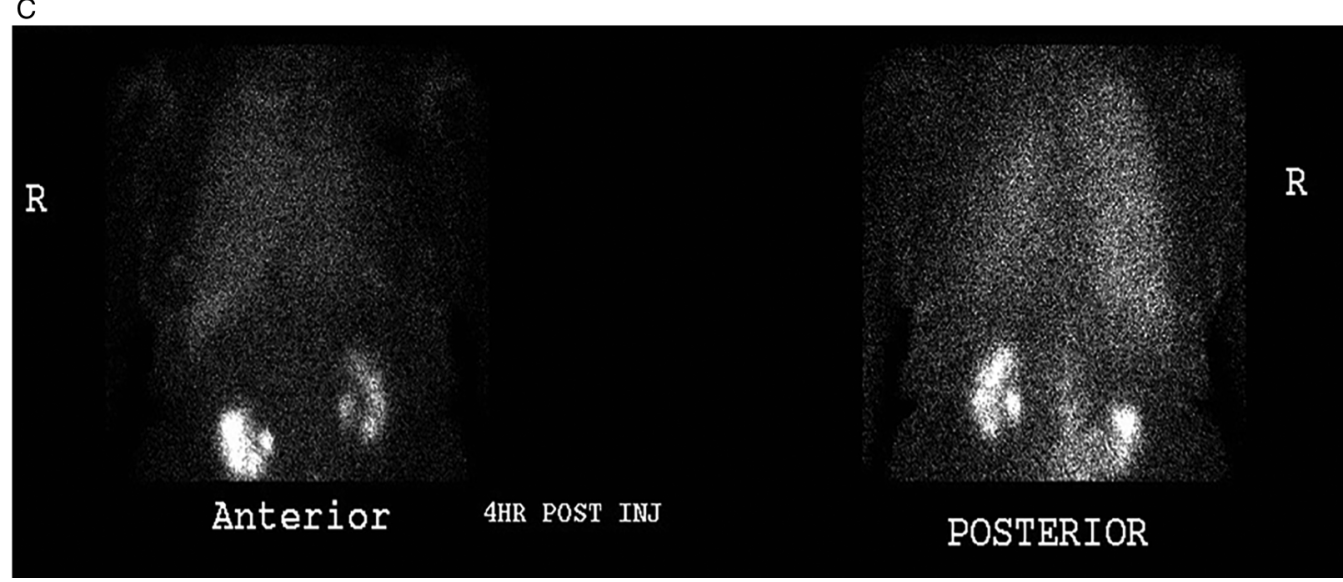

Figure 1 (A) Chest radiograph, 'posteroanterior view', showing right-sided moderate pleural effusion. (B) CT scan reveals visceral pleural thickening with small loculated basilar pneumothorax from trapped lung. (C) Technetium 99m renal perfusion scintigram scan reveals extravasation of the contrast material from the abdominal cavity into the right pleural space.
To cite: Chopra A,
Argula R, Schaefer $C$, et al. Thorax 2016;71:10641065. 


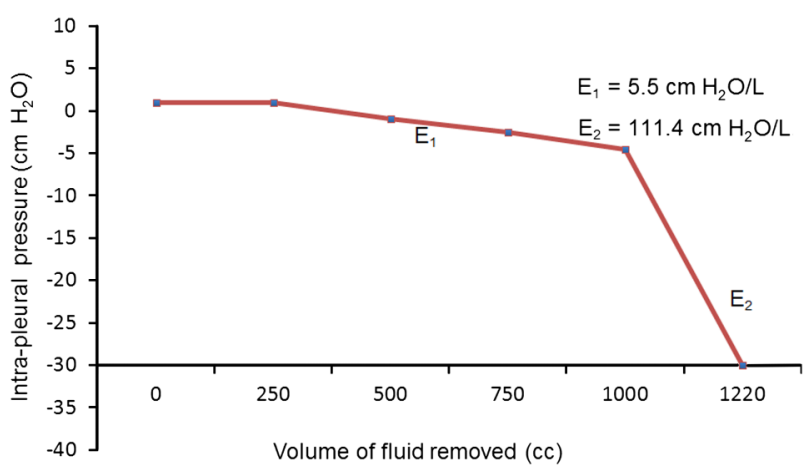

Figure 2 The pleural pressure-volume curve demonstrating a biphasic slopes. $E_{1}$ denotes the normal pleural elastance curve before the inflection point (0). Continued aspiration beyond the inflection point demonstrates a steep decline in the intrapleural pressure due to an abnormally high pleural space elastance $\left(E_{2}\right)$ from visceral pleural restriction consistent with trapped lung.

\section{DISCUSSION}

The diagnosis of pleural transudates could be challenging, especially when competing mechanisms could be causing pleural fluid formation and persistence. Our patient is a good example of a 'dual diagnosis'; that of an urinothorax in the presence of a pre-existing trapped lung from remote haemothorax. Our case also illustrates the clinical value of pleural manometry and careful preprocedural ultrasound scanning in rendering the diagnoses at bedside.
Using pleural manometry, we demonstrated a biphasic pleural elastance (Pel) curve (figure 2). During the initial phase of the thoracentesis, the patient had a normal Pel, consistent with an expandable lung (E1). After drainage of approximately $1 \mathrm{~L}$ of fluid, there was steep decrease in pleural pressure (E2) indicating a sharp increase in Pel. This P-V curve is indicative of an unexpandable lung. A biphasic P-V curve is commonly seen in the presence of lung entrapment due to either malignancy or complicated parapneumonic effusion. ${ }^{1}{ }^{2}$ However, the presence of a biphasic P-V curve in a patient with a pre-existing transudate can only occur as a result of a dual mechanism, with one cause being a trapped lung and another process resulting in the collection of more transudative fluid in the pleural space. ${ }^{2}$ Careful attention to subdiaphragmatic structures during preprocedural ultrasonographic evaluation, such as the kidney in our patient, may provide a useful diagnostic information with regard to the aetiology of pleural effusion.

Contributors $\mathrm{TH}$ is the guarantor of the paper, and takes responsibility for the integrity of the work as a whole, from inception to published article. All authors contributed to the writing of the manuscript.

Competing interests None declared.

Patient consent Obtained.

Provenance and peer review Not commissioned; externally peer reviewed.

\section{REFERENCES}

1 Huggins JT, Doelken P, Sahn SA. The unexpandable lung. F1000 Med Rep 2010;2:77.

2 Huggins JT, Sahn SA, Heidecker J, et al. Characterisitics of trapped lung: pleural fluid analysis, manometry, and air-contrast chest CT. Chest 2007;131:206-13. 